\title{
Trigonal to Pentagonal Bipyramidal Coordination Switching in a Co(II) Single-Ion Magnet
}

\author{
Moya A. Hay, ${ }^{\dagger}$ Charles J. McMonagle, ${ }^{\ddagger}$ Claire Wilson, ${ }^{\dagger}$ Michael R. Probert, ${ }^{\ddagger}$ and Mark Murrie ${ }^{*}{ }^{\dagger}(0)$ \\ ${ }^{\dagger}$ WestCHEM, School of Chemistry, University of Glasgow, Glasgow, G12 8QQ United Kingdom \\ ${ }^{\ddagger}$ Chemistry, School of Natural and Environmental Sciences, Newcastle University, Newcastle upon Tyne, NE1 7RU, United \\ Kingdom
}

Supporting Information

ABSTRACT: In molecular magnetism and single-ion magnets in particular, the observation of slow relaxation of the magnetization is intimately linked to the coordination environment of the metal center. Such systems typically have blocking temperatures well below that of liquid nitrogen, and therefore detailed magnetic characterization is usually carried out at very low temperatures. Despite this, there has been little advantage taken of ultralow temperature single-crystal X-ray diffraction techniques that could provide a full understanding of the crystal structure in the same temperature regime where slow magnetic relaxation occurs. Here, we present a systematic variable temperature single crystal X-ray

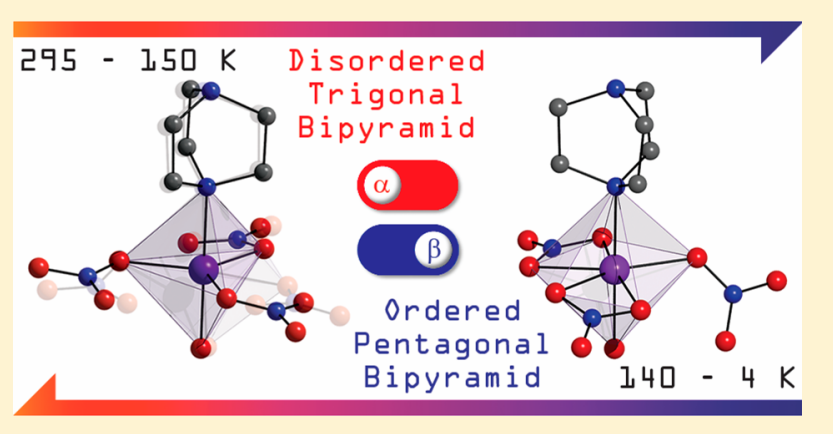
diffraction study of $\left[\mathrm{Co}^{\mathrm{II}}\left(\mathrm{NO}_{3}\right)_{3}\left(\mathrm{H}_{2} \mathrm{O}\right)(\mathrm{HDABCO})\right]$ (1)

$\{\mathrm{DABCO}=1,4$-diazabicyclo[2.2.2] octane $\}$ conducted between 295 to $4 \mathrm{~K}$. A reversible and robust disorder-to-order, singlecrystal to single-crystal phase transition was identified, which accompanied a switching of the coordination geometry around the central Co(II) from 5- to 7-coordinate below $140 \mathrm{~K}$. The magnetic properties were investigated, revealing slow relaxation of the magnetization arising from a large easy-plane magnetic anisotropy $(+D)$ in the $\mathrm{Co}$ (II) pentagonal bipyramidal environment observed at low temperatures. This study highlights the importance of conducting thorough low temperature crystallographic studies, particularly where magnetic characterization is carried out at such low temperatures.

\section{INTRODUCTION}

Cobalt is widely used in chemistry, from biological applications such as antibacterial and therapeutic agents to electrochemical catalysis and molecular magnetism. ${ }^{1-4}$ This diverse range of applications reflects cobalt's rich and interesting behavior, which stems from extensive redox, optical, and magnetic properties. In a molecular magnetism context, $\mathrm{Co}$ (II)-based complexes are particularly attractive to study as they can show slow relaxation of the magnetization in a range of coordination environments. Co(II) can help address two requirements for successful single-molecule magnets (SMMs) - a significant spin-orbit coupling (SOC) contribution to the magnetic anisotropy (first or second order depending on geometry) and control over undesirable relaxation processes. ${ }^{5-11}$ The latter is a result of the Kramers Theorem, where quantum tunneling of the magnetization (QTM) and direct spin-phonon relaxation between the ground state Kramers doublet is formally forbidden for the half-integer spin $\mathrm{Co}$ (II) ion, regardless of the sign of the axial zero-field splitting (ZFS) parameter $D{ }^{12-20}$ Both of these factors contribute to the success of $\mathrm{Co}(\mathrm{II})$ in polynuclear $3 \mathrm{~d}, 3 \mathrm{~d}-4 \mathrm{f}$, and mononuclear $3 \mathrm{~d}$ cobalt SMMs. ${ }^{21-26}$ For mononuclear Co(II) complexes, the focus is firmly on enhancing the axial magnetic anisotropy of the system. $^{27}$ However, in order to understand the magnetic anisotropy in $\mathrm{Co}$ (II) mononuclear complexes, which tends to dictate the resulting magnetic properties, a thorough understanding of the geometrical environment of the $\mathrm{Co}(\mathrm{II})$ is required. ${ }^{28}$ In particular, where low temperature magnetic measurements are used to confirm certain properties, it is also important to be certain of the structure in the temperature range at which the measurement is carried out (typically $<10$ K). ${ }^{29}$

We present herein a systematic variable temperature single crystal X-ray diffraction study of $\left[\mathrm{Co}^{\mathrm{II}}\left(\mathrm{NO}_{3}\right)_{3}\left(\mathrm{H}_{2} \mathrm{O}\right)\right.$ ( $\mathrm{HDABCO})](\mathbf{1}),{ }^{30}$ evidencing a disorder-to-order, singlecrystal to single-crystal phase transition accompanied by a clear coordination switch from a 5- to a 7-coordinate complex below $140 \mathrm{~K}$. The magnetic studies of 1 confirm slow relaxation of the magnetization under an applied dc field, originating from a large easy-plane magnetic anisotropy. We stress the merits of thorough low temperature crystallographic investigations of single-ion magnets, especially where the ligands involved can display a variety of coordination modes.

\section{EXPERIMENTAL SECTION}

Physical Methods. Elemental analysis (EA) was performed inhouse by the microanalysis service at the School of Chemistry,

Received: February 25, 2019

Published: July 22, 2019 
University of Glasgow. IR spectra were collected using a Shimadzu FTIR spectrometer in the range $4000-600 \mathrm{~cm}^{-1}$. Single-crystal X-ray diffraction data were collected for complex 1 under ambient conditions and at regular intervals down to $4 \mathrm{~K}$ at Newcastle University. Data from 295 to $80 \mathrm{~K}$ were collected using a Bruker D8 VENTURE diffractometer (Ag $\mathrm{K} \alpha$ radiation, $\lambda=0.56087 \AA$ ) equipped with a Photon II CPAD detector and an Oxford Cryosystems Cryostream 800 device. SAINT ${ }^{31}$ was used to integrate the data, and empirical absorption corrections using equivalent reflections were performed with the program SADABS. ${ }^{32}$ Data from 100 to $4 \mathrm{~K}$ were collected by mounting the sample on a graphite rod which was placed inside the cryostat of the XIPHOS I diffractometer (Mo $\mathrm{K} \alpha$ radiation, $\lambda=0.71073 \AA$ ). ${ }^{33,34}$ The system has a minimum operating temperature of $1.86 \mathrm{~K}$, achieved using a modified two-stage closed-cycle refrigerator enhanced with an additional third JouleThompson stage. Ambient temperature data were collected before and after cooling. Data were integrated using SAINT, ${ }^{31}$ and empirical absorption corrections using equivalent reflections were performed with the program SADABS. ${ }^{32}$ Crystallographic data for complex 2 at $100 \mathrm{~K}$ were collected by the National Crystallography Service (NCS). For data collected at $295 \mathrm{~K}$, a Bruker D8 VENTURE diffractometer equipped with a Photon II CMOS detector with an Oxford Cryosystems N-Helix device mounted on a dual $\mathrm{Cu}$ and $\mathrm{Mo} \mathrm{I} \mu \mathrm{S}$ 3.0 microfocus sealed tube generator was used. Structures were solved using SHELXT and refined using SHELXL full-matrix least-squares refinement within Olex $^{2}$ software. ${ }^{35,36}$ The powder X-ray patterns were collected on a PANalytical XPert MPD, with $\mathrm{Cu} \mathrm{K} \alpha$ radiation at ambient temperature over a range of $5^{\circ}<2 \theta<50^{\circ}$ using a step size of $0.0167^{\circ}$. The calculated pattern was generated from Mercury using the CIF of the crystal structure at room temperature. ${ }^{37}$ All magnetic measurements were carried out on powdered crystalline samples restrained in eicosane using a Quantum Design MPMS-XL SQUID magnetometer. The data were corrected for the diamagnetic contribution of the sample holder and eicosane by measurements, and for the diamagnetism of compound $1\left(\chi_{\text {DIA }}=1.88 \times 10^{-4} \mathrm{~cm}^{3}\right.$. $\left.\mathrm{mol}^{-1}\right)$ and $2\left(\chi_{\text {DIA }}=1.91 \times 10^{-4} \mathrm{~cm}^{3} \cdot \mathrm{mol}^{-1}\right)$.

Synthetic Methods. All reagents and solvents were obtained commercially and used without any further purification. The ligand 1,4-diazabicyclo[2.2.2] octane (DABCO) is commercially available and was used as obtained. For the synthesis of $\mathrm{Zn}_{x} \mathrm{Co}_{1-x}\left(\mathrm{NO}_{3}\right)_{3}\left(\mathrm{OH}_{2}\right)(\mathrm{HDABCO})$ (2), see the Supporting Information.

[Co $\left.\left(\mathrm{NO}_{3}\right)_{3}\left(\mathrm{OH}_{2}\right)(\mathrm{HDABCO})\right](1) . \mathrm{Co}\left(\mathrm{NO}_{3}\right)_{2} \cdot \mathrm{H}_{2} \mathrm{O}(0.58 \mathrm{~g}, 2 \mathrm{mmol})$ was stirred in a 1:3 mixture of $\mathrm{MeCN}$ and $\mathrm{MeOH}(20 \mathrm{~mL})$ resulting in a pink-orange solution. DABCO $(0.22 \mathrm{~g}, 2 \mathrm{mmol})$ was added and the pink suspension stirred at approximately $60{ }^{\circ} \mathrm{C}$ for $2 \mathrm{~h}$. The final purple suspension was filtered giving a purple precipitate and bright pink solution, which, on vapor liquid diffusion with diethyl ether, yielded pink plate crystals after 1 day suitable for single crystal X-ray diffraction. The purple precipitate was later discarded as no pure product could be obtained on redissolving. Yield: $8 \%(61 \mathrm{mg})$. IR ( $\nu$ in $\left.\mathrm{cm}^{-1}\right)$ : $3433(\mathrm{w}), 3179(\mathrm{w}), 1635(\mathrm{w}), 1442(\mathrm{~s}), 1288(\mathrm{~s}), 1206$ $(\mathrm{s}), 1029(\mathrm{~s}), 849(\mathrm{~m}), 794(\mathrm{~s}), 748(\mathrm{w}), 617(\mathrm{~m}) . \mathrm{EA}$ analysis $\left(\mathrm{CoC}_{6} \mathrm{H}_{15} \mathrm{~N}_{5} \mathrm{O}_{10} \cdot 0.25 \mathrm{CH}_{4} \mathrm{O}\right)$ [\%], found: $\mathrm{C}, 19.42 ; \mathrm{H}, 4.07 ; \mathrm{N}, 18.15$. Calcd: C, 19.54; H, 4.20; N, 18.2.

\section{RESULTS AND DISCUSSION}

Crystal Structure Description. [CO $\left(\mathrm{NO}_{3}\right)_{3}\left(\mathrm{OH}_{2}\right)-$ (HDABCO)] $-\alpha$ Phase (High Temperature). Single crystal Xray data collected at ambient temperature revealed that $\mathbf{1}$ crystallizes in the monoclinic $P 2_{1}$ space group (see Table S1 for crystallographic data). The asymmetric unit consists of a full molecule of $\mathbf{1}$, which comprises a central $\mathrm{Co}$ (II) ion with a protonated $[\mathrm{HDABCO}]^{+}$ligand coordinated in one axial position, and a $\mathrm{H}_{2} \mathrm{O}$ molecule coordinated in the other (see Figure 1). Three symmetrically distinct monodentate $\mathrm{NO}_{3}{ }^{-}$ $\left(\eta^{1}-\mathrm{ONO}_{2}\right)$ ligands in the equatorial positions complete the coordination sphere. The nitrate at the N5 position exhibits

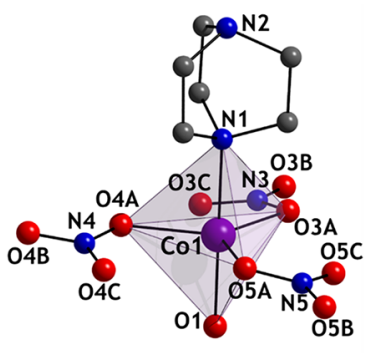

Trigonal bipyramidal (1) $75 \%$

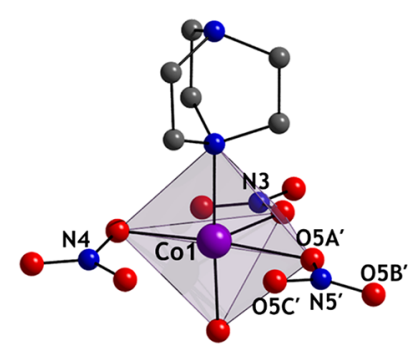

Vacant octahedron (1')
Figure 1. Asymmetric unit of complex 1 and $\mathbf{1}^{\prime}$ at ambient temperature $(295 \mathrm{~K})$ with respective polyhedra (TBP and vacant octahedron) depicted. Hydrogen atoms have been omitted for clarity.

disorder between two different positions under ambient conditions. This disorder leads to two distinct 5-coordinate geometries around the Co(II) center such that, for clarity, we will discuss the disorder in terms of two separate complexes$\mathbf{1}$ and $\mathbf{1}^{\prime}$ (see Figure 1). At the N5 position of $\mathbf{1}$, and the N5' position of $1^{\prime}$, the $\eta^{1}-\mathrm{ONO}_{2}$ was refined competitively to 0.74539 and 0.25461 , respectively. This means that the position of the $\eta^{1}-\mathrm{ONO}_{2}$ at the N5 position in $\mathbf{1}$ is adopted within the crystal lattice approximately $75 \%$ of the time and $25 \%$ of the time, that at the $\mathrm{N}^{\prime}$ in $\mathbf{1}^{\prime}$. The positioning of the $\eta^{1}-\mathrm{ONO}_{2}$ at the $\mathrm{N} 5$ and $\mathrm{N}^{\prime}$ positions in $\mathbf{1}$ and $\mathbf{1}^{\prime}$ results in a trigonal bipyramidal and a square pyramidal like geometry, respectively (see Figure 1). The closest geometries were determined via SHAPE studies confirming a trigonal bipyramidal coordination environment for $\mathbf{1}$ and a vacant octahedron for $\mathbf{1}^{\prime}$, with $\mathrm{O} 4 \mathrm{C}$ orientated in the vacant position (see Tables S4 and S5). ${ }^{38-40}$ Single crystal X-ray diffraction data sets were measured at predetermined intervals between 295 and $150 \mathrm{~K}$ to confirm what, if any, effect cooling down 1 would have on the observed disorder (see Table S1 for crystallographic data). The occupancy of the disordered nitrate for 1 steadily increased $(75 \% \rightarrow 95 \%)$ accompanied by the appropriate decrease in occupation for $\mathbf{1}^{\prime}(25 \% \rightarrow 5 \%)$ on approaching $150 \mathrm{~K}$, indicating that the disorder is dynamic in nature (see Figure S3). Below $150 \mathrm{~K}$, a structural phase transition is observed, and this will be discussed in more detail later. Two molecules make up the unit cells of $\mathbf{1}$ and $\mathbf{1}^{\prime}$. Hydrogen bonding interactions propagate along both the $a$ and $c$ axes of the lattice between the coordinated water molecule to nitrates on adjacent molecules $\left(\mathrm{O} 1-\mathrm{H} 1 \mathrm{~A} \cdots \mathrm{O} \mathrm{B}^{i}\right.$ and $\mathrm{O} 1-$ $\left.\mathrm{H} 1 \mathrm{~B} \cdots \mathrm{O}_{3} \mathrm{~B}^{i i}(i=x, y, z-1 ; i i=x-1, y, z)\right)$ with distances of $1.8326(2)$ and 2.0491(3) $\AA$, respectively, at $150 \mathrm{~K}$. The molecules of $\mathbf{1}$ and $\mathbf{1}^{\prime}$ align in rows in the $a$ and $c$ directions of the crystal lattice (see Figure S2), with the shortest intermolecular Co..Co distances of 6.8758(10) $\AA$ (from 150 $\mathrm{K}$ structure) found along the $a$ axis. The longest intermolecular Co..Co distances in the lattice occur between molecules of different rows, or between molecules along the $b$ axis, at $8.4725(8) \AA$ (at $150 \mathrm{~K})$.

$\left[\mathrm{Co}\left(\mathrm{NO}_{3}\right)_{3}\left(\mathrm{OH}_{2}\right)(\mathrm{HDABCO})\right]-\beta$ Phase (Low Temperature). On cooling below $140 \mathrm{~K}$, a disorder-to-order, single-crystal to single-crystal transformation is observed where cooling the system results in reorganization of the nitrate ligands around the $\mathrm{Co}(\mathrm{II})$ center (see Figure 2). The transformation is reversible and robust, and on heating the sample to $295 \mathrm{~K}$, the $\alpha$ phase can be obtained once again without a loss in 


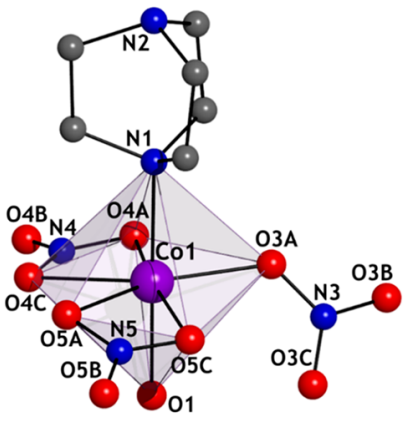

Figure 2. Asymmetric unit of complex 1 after cooling below $140 \mathrm{~K}$ with the pentagonal bipyramidal coordination polyhedron depicted. Hydrogen atoms have been omitted for clarity.

crystallinity. Although the $\alpha$ (high temperature) and $\beta$ (low temperature) phases crystallize in the same space group, this phase transition is accompanied by a change in the unit cell volume and $\beta$ angle lattice parameter as shown in Figure 3. Further crystallographic data were collected at predetermined intervals from 140 to $4 \mathrm{~K}$ to observe if there would be any further structural changes; however, this was not observed, and the $\beta$ phase is retained. Crystal data and structure refinement parameters for these collections can be found in the Supporting Information, Tables S2 and S3. On the transition from the $\alpha$ to the $\beta$ phase, the once disordered $\eta^{1}-\mathrm{ONO}_{2}$ at the N5 position of complexes $1 / \mathbf{1}^{\prime}$ becomes ordered with an accompanied change in coordination mode from mono- to bidentate $\left(\eta^{2}\right.$-OONO). Although the nitrate at the $\mathrm{N} 4$ position did not exhibit disorder in the $\alpha$ phase, the coordination mode has also changed from mono- to bidentate. The nitrate at the $\mathrm{N} 3$ position remains monodentate but drops down and orients toward the water molecule coordinated in the axial position. Thus, the Co(II) center of complex 1 is 7 -coordinate in the $\beta$ phase and adopts a pentagonal bipyramid geometry as confirmed via SHAPE studies (see Table S7). ${ }^{38-40}$ However, the $\alpha \rightarrow \beta$ phase transition is not accompanied by drastic changes in the unit cell parameters (cf. 150 and $140 \mathrm{~K}$ crystallographic data presented in Tables S1 and S2) or in the crystal packing of the molecules within the lattice. A hydrogen bonding interaction again propagates along the $a$ axis between the coordinated water molecule to nitrates on adjacent molecules $\left(\mathrm{O} 1-\mathrm{H} 1 \mathrm{~A} \cdot \mathrm{O}^{\mathrm{O}} 3 \mathrm{~B}^{i}(i=x-1, y, z)\right)$ with a distance of 2.0364(3) $\AA$ (at $140 \mathrm{~K}$ ). However, in the case of the $\beta$ phase of $\mathbf{1}$, there is no interaction propagating along the $c$ axis as observed with the $\alpha$ phase. The molecules of $\mathbf{1}$ again align in rows in the a direction of the crystal lattice (see Figure S4) with the shortest intermolecular $\mathrm{Co} \cdot \mathrm{Co}$ distances of $6.8106(11) \AA$ (at $140 \mathrm{~K}$ ) along this axis, and the longest Co..Co distances of $8.5955(9) \AA$ (from $150 \mathrm{~K}$ structure) arising between molecules along the $b$ axis.

Magnetic Properties. The variable temperature magnetic susceptibility data of complex 1 were collected between 290 and $2 \mathrm{~K}$ under an applied direct-current $(\mathrm{dc}$ ) field of $0.1 \mathrm{~T}$ (see Figure 4). The measurement was performed on pure single crystals that were ground to a fine powder and then restrained in eicosane to prevent reorientation and torqueing. At $290 \mathrm{~K}$, the $\chi_{\mathrm{m}} T$ value for 1 is $2.74 \mathrm{~cm}^{3} \mathrm{~mol}^{-1} \mathrm{~K}$. This value is higher than the theoretical value of $2.27 \mathrm{~cm}^{3} \mathrm{~mol}^{-1} \mathrm{~K}$ where $S=3 / 2$ and $g=2.2$ are calculated using the spin-only formula, indicating a significant orbital contribution to the magnetic susceptibility. The $\chi_{\mathrm{m}} T$ vs $T$ data slowly decrease until $160 \mathrm{~K}$ is reached, after which a faster drop in $\chi_{\mathrm{m}} T$ is observed, steadying again after $140 \mathrm{~K}$ (see Figure 4 and inset). This could be due to the phase transition $(\alpha \rightarrow \beta)$ observed in the $\mathrm{X}$-ray diffraction studies, arising from the change from a trigonal bipyramidal to a pentagonal bipyramidal geometry. However, it is not surprising that the difference in the $\chi_{\mathrm{m}} T$ data around the temperature of the phase transition is not particularly prominent based on previous measurements conducted on TBP and PBP Co(II) complexes reported in the literature. ${ }^{9,10,41-45}$ Furthermore, this feature would be quite difficult to spot in the absence of the variable temperature Xray study, which would lead to an erroneous analysis of the low temperature magnetic data as arising from a trigonal bipyramidal Co(II) complex. The $\chi_{\mathrm{m}} T$ vs $T$ data then gradually decrease until $40 \mathrm{~K}$ is reached, after which a sharp maximum is observed with $\chi_{\mathrm{m}} T_{\max }=3.29 \mathrm{~cm}^{3} \mathrm{~mol}^{-1} \mathrm{~K}$ observed at $12 \mathrm{~K}$. The $\chi_{\mathrm{m}} T$ value then rapidly drops with $\chi_{\mathrm{m}} T_{\min }=2.09 \mathrm{~cm}^{3}$ $\mathrm{mol}^{-1} \mathrm{~K}$ recorded at $2 \mathrm{~K}$. In the low temperature $\beta$ phase of $\mathbf{1}$, the shortest intermolecular Co..Co distance is $\sim 6.8 \AA$, where there is a hydrogen bonding interaction between a coordinated water molecule and a nitrate ligand on an adjacent molecule, $\left\{\mathrm{Co}-\mathrm{H}_{2} \mathrm{O} \cdots \mathrm{NO}_{3}-\mathrm{Co}\right\}$. The similar $\mathrm{Cu}-\mathrm{H}_{2} \mathrm{O} \cdots \mathrm{NO}_{3}-\mathrm{Cu}$ motif has been observed to transmit exchange interactions between $\mathrm{Cu}$ (II) centers $(\sim 7 \AA$ apart $)$ in $\left\{\mathrm{Cu}\left(\mathrm{NO}_{3}\right)\left(\mathrm{H}_{2} \mathrm{O}\right)\right\}$ $(\mathrm{HTae})(\mathrm{Bpy})\left(\mathrm{H}_{2} \mathrm{Tae}=1,1,2,2\right.$-tetraacetylethane, $\mathrm{Bpy}=4,4^{\prime}$ bipyridine). ${ }^{46}$ To probe this further, we attempted the synthesis of a magnetically dilute form of complex 1 using
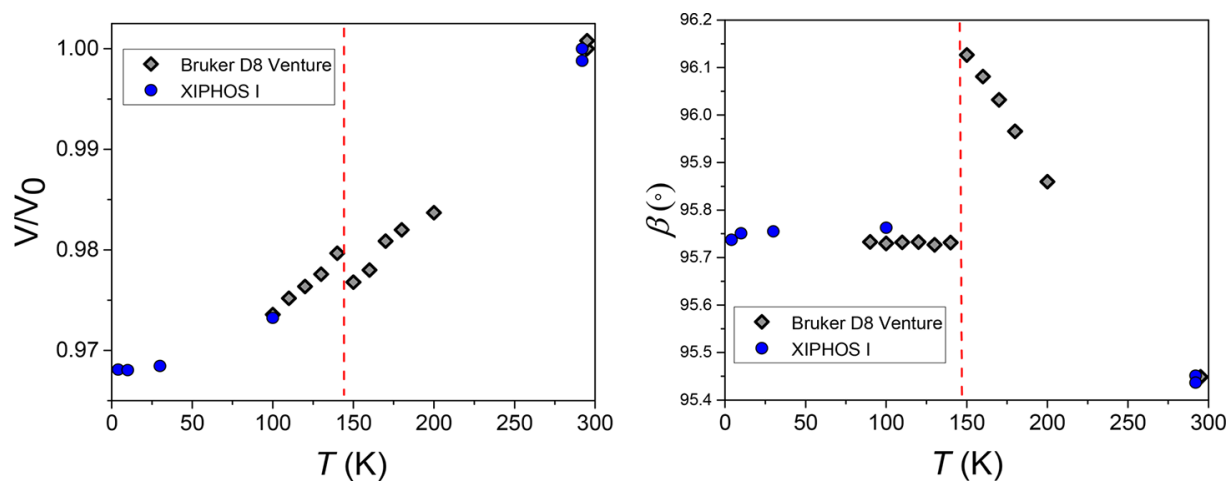

Figure 3. Temperature dependence of the normalized unit cell volume $V / V_{0}$ (left) and of the $\beta$ angle lattice parameter (right) with the phase transition marked with a red dashed line. Data collected on the Bruker D8 Venture are presented as gray diamonds, and data collected on the XIPHOS I are shown as blue diamonds and show reasonable agreement. 

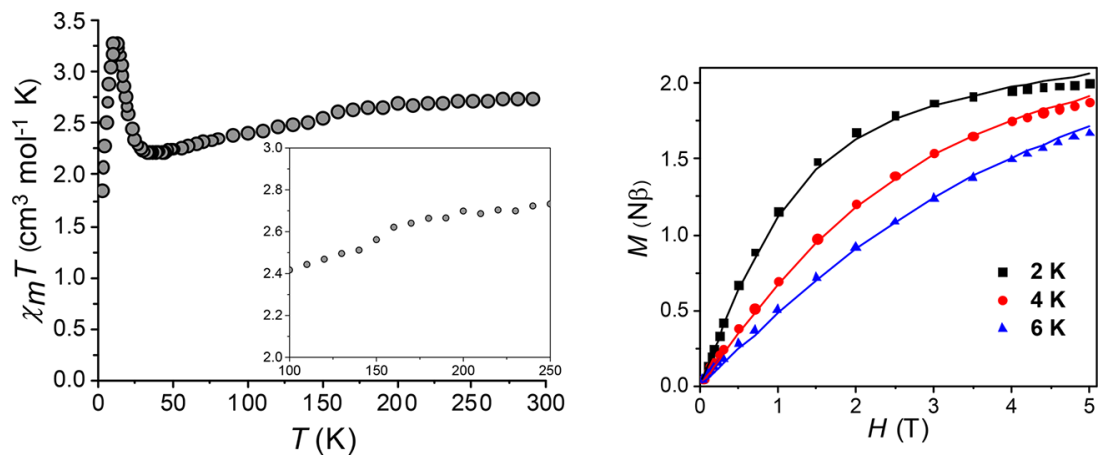

Figure 4. Temperature dependence of $\chi_{\mathrm{m}} T$ for 1 from 290 to $2 \mathrm{~K}$ at $0.1 \mathrm{~T}$ with the inset showing the region between 250 and $100 \mathrm{~K}$ in more detail (left) and the magnetization versus field curves at 2,4 , and $6 \mathrm{~K}$ (right). The solid lines correspond to the fit of the data (see text for details).

$\mathrm{Zn}(\mathrm{II})-\left[\mathrm{Zn}_{x} \mathrm{Co}_{1-x}\left(\mathrm{NO}_{3}\right)_{3}\left(\mathrm{H}_{2} \mathrm{O}\right)(\mathrm{HDABCO})\right]$ (2) (see Supporting Information for crystallographic data and details of synthesis). Although complex 2 was successfully prepared, the $\alpha \rightarrow \beta$ phase transition was not seen in the crystallographic data, and a trigonal bipyramidal complex was instead obtained, in agreement with SHAPE studies (see Figure S6 and Table S9). Although it is not entirely appropriate to compare the low temperature $\chi_{\mathrm{m}} T$ vs $T$ data for 1 , where the $\mathrm{Co}$ (II) resides in a PBP coordination environment, to that of 2 , where $\mathrm{Co}$ (II) is in a TBP environment, the magnetically dilute complex 2 does not exhibit the low temperature feature in $\chi_{\mathrm{m}} T$ vs $T$ (see Figure S6). Variable field magnetization measurements ( $M$ vs $H$ ) were carried out on 1 and 2 between 0 and $5 \mathrm{~T}$ at 2, 4, and $6 \mathrm{~K}$ (see Figure 4 and S6), and in both cases, the magnetization does not reach saturation, indicative of a large magnetic anisotropy.

To obtain information on the zero-field splitting (ZFS) parameters, the $M$ vs $H$ data were fitted for 1 using the program $P H I$ in accordance with the following expression: ${ }^{47}$

$$
\hat{H}=D \hat{S}_{z}^{2}+E\left(\hat{S}_{x}^{2}-\hat{S}_{y}^{2}\right)+\mu_{\mathrm{B}} \vec{B} \cdot \overleftrightarrow{g} \cdot \hat{S}
$$

where the first and second terms describe the axial and rhombic ZFS interactions parametrized through $D$ and $E$, respectively. The third term takes into account the Zeeman interaction with the spin operator $\hat{S}$, applied field $B$, and Landé tensor $g$. Fits can be carried out using both the $\chi_{\mathrm{m}} T$ vs $T$ and $M$ vs $H$ data sets simultaneously; however, we carried out the fit using just the $M$ vs $H$ data for $\mathbf{1}$ for three reasons. First, we wanted to avoid taking into account the low temperature feature in the $\chi_{\mathrm{m}} T$ vs $T$ data. Second, the $\chi_{\mathrm{m}} T$ vs $T$ data exhibit a small "dip" around the phase transition $(\alpha \rightarrow \beta)$ temperature. Both of these features could add to uncertainty in the parameters obtained from the fit. Third, we want to obtain parameters relating only to the low temperature $(\beta)$ phase, where the $\mathrm{Co}$ (II) resides in a pentagonal bipyramidal coordination geometry.

To obtain initial starting values for the fit, a survey was conducted using PHI (see Figure S7). ${ }^{47}$ This involved surveying both $D$ and $g$ to identify a local minimum in the residual. A minimum was identified at $g=2.30$ and $D=+31.9$ $\mathrm{cm}^{-1}$. Both the sign and magnitude of $D$ are very reasonable considering parameters reported for other hepta-coordinate Co(II) structures (see Table S8). Using $g$ and $D$ as fixed parameters, $E / D=0.16$ was then obtained from the fit, with a residual of $99.3 \%$. The $E / D$ parameter is larger than that of other related complexes; however, on comparison of the CShM values, complex $\mathbf{1}$ is found to be significantly more distorted. $^{42,43,48}$
Alternating current (ac) susceptibility measurements were carried out to investigate the magnetic relaxation dynamics of 1. Initially, a sweep of the temperature between 10 and $2 \mathrm{~K}$ in both zero-applied field and under $H_{\mathrm{dc}}=0.2 \mathrm{~T}$ was performed (see Figure S8). Complex 1 exhibits a response in both cases. However, the behavior in zero and applied fields differs with the maxima in the out-of-phase response appearing at high and low temperatures, respectively. Therefore, more extensive ac susceptibility measurements were carried out to investigate the magnetic relaxation dynamics of $\mathbf{1}$ under both conditions.

In the absence of an applied field, variable temperature (20$2 \mathrm{~K}$ ) ac susceptibility measurements were performed on $\mathbf{1}$ with the in-phase $\left(\chi^{\prime}\right)$ and out-of-phase $\left(\chi^{\prime \prime}\right)$ data shown as a function of frequency $(1-1488 \mathrm{~Hz})$ in Figure S9. Although these data are reminiscent of a spin-glass transition, ${ }^{49,50}$ given the crystal structure of $\mathbf{1}$, this unusual behavior is more likely to arise from a small unidentified impurity phase not visible in the PXRD data. As this behavior can be suppressed in the presence of an applied dc field (Figure S8), the field dependence of the ac susceptibility was investigated in order to determine the optimum field at which to probe the temperature dependence of the ac susceptibility for 1 . The variable field measurements $(0.05-0.5 \mathrm{~T})$ at a constant temperature of $2 \mathrm{~K}$ were carried out (see Figure S11). A fit of the field-dependent ac susceptibility data was extracted from the Argand diagram $\left(\chi^{\prime}\right.$ vs $\left.\chi^{\prime \prime}\right)$ using the program CC-Fit. (Figure S12, left). ${ }^{51,52}$ As the optimum $\chi^{\prime \prime}$ response (where relaxation of magnetization is slowest) is observed at $H_{\mathrm{dc}}=$ 1000 Oe (Figure S12, right), variable temperature ac susceptibility studies were conducted under this field between 1.8 and $8 \mathrm{~K}$ (see Figure 5). A clear out-of-phase signal is observed for $\mathbf{1}$, with the maxima lying beyond the frequency accessible limit above $3 \mathrm{~K}$. The ac susceptibility data were fitted using the program CC-Fit and, in accordance with the generalized Debye model, the relaxation rates $\tau$ for each temperature extracted (see Figure S13). ${ }^{51,52}$ The subsequent Arrhenius plot ( $\ln \tau$ vs $1 / T$ ) exhibits a crossover between relaxation regimes, where the dominant relaxation pathways move from a thermal to a quantum regime (see Figure S14).

A linear fit of the $\ln \tau$ vs $1 / T$ data in the high temperature region $(4.5-7 \mathrm{~K})$ to account for relaxation via thermally activated pathways was performed in accordance with eq 2 , where the gradient gives a value for the effective energy barrier $\left(U_{\text {eff }}\right)$. Parameters obtained from the fit were $U_{\text {eff }}=39.0 \pm 2.6$ $\mathrm{K}\left(27.1 \pm 1.8 \mathrm{~cm}^{-1}\right)$ and the pre-exponential factor $\tau_{0}=6.51 \pm$ $0.62 \times 10^{-9} \mathrm{~s}$. This barrier is too large to arise from a preferred axis within the $x y$ plane due to the rhombic anisotropy. 


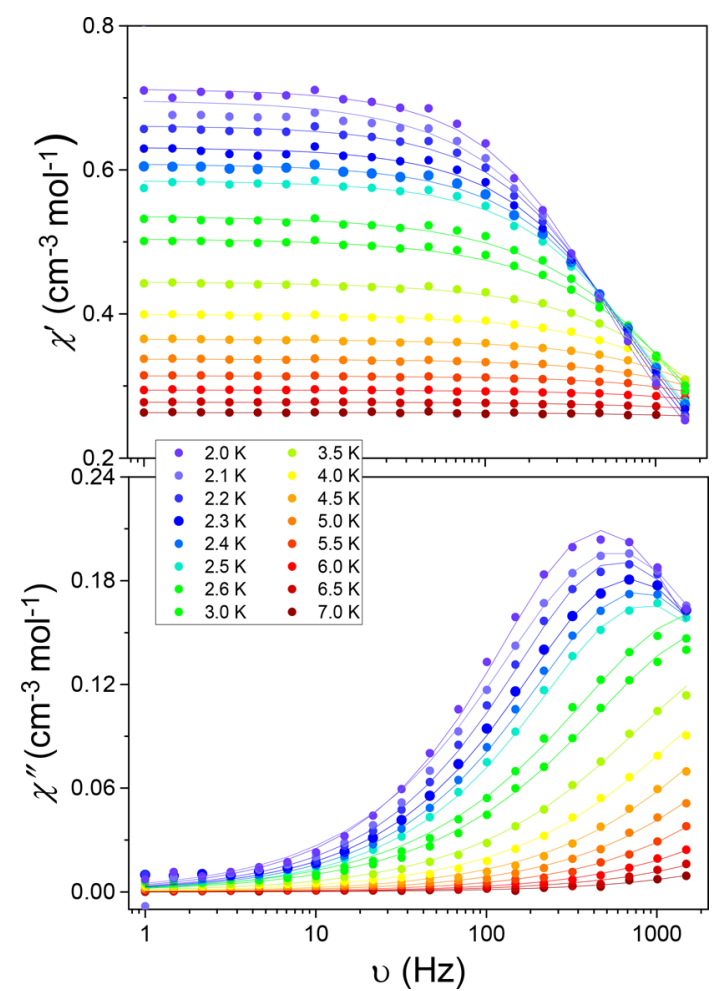

Figure 5. Frequency-dependence of the in-phase $\left(\chi^{\prime}\right)$ and out-ofphase $\left(\chi^{\prime \prime}\right)$ signal for complex 1 over a range of temperatures in an applied field $H_{\mathrm{dc}}=1000$ Oe.

Alternatively, for a $\mathrm{Co}$ (II) system with a positive $D$ parameter, Orbach relaxation via the $m_{\mathrm{s}}= \pm 3 / 2$ states can occur if direct relaxation between the $m_{\mathrm{s}}= \pm 1 / 2$ states is slow. However, from the approximation $U_{\text {eff }} \sim 2|D|$ and results of the $\mathrm{dc}$ magnetic data fit, the energy barrier should be $\sim 62 \mathrm{~cm}^{-1}$, and hence, the $U_{\text {eff }}$ value obtained is less than half of that predicted. This is common for easy-axis $(+D)$ systems with small $E$ terms, where relaxation is mediated instead via the hyperfine interaction of the nuclear spin and molecular vibrations. ${ }^{20,53-55}$ Therefore, the fit was reconsidered using the extended Debye model shown in eq 3 (see Figure S15). This model takes into account the spin-lattice relaxation Raman $\left(\tau \propto T^{\mathrm{n}}\right)$ processes, as well as quantum tunneling of magnetization (QTM) given as the first and second terms, respectively. ${ }^{9,56,57}$ The two phonon Raman process $\left(\propto T^{n}\right)$ was found to be dominant in the high temperature region, with $C=0.23(0.05) \mathrm{s}^{-1} \mathrm{~K}^{-n}$ and $n=7.65(0.11)$ obtained from the fit. Although $n$ should equal 9 for a Kramers ion, this value may be lower if optical and acoustic phonons are taken into account. ${ }^{58-61}$ Inclusion of the term relating to a QTM relaxation pathway was necessary to fit the data $\left(\mathrm{QTM}=5.92(1.50) \times 10^{-3} \mathrm{~s}^{-1}\right)$, as expected given the indication of quantum and thermally independent processes in the Arrhenius plot.

$$
\begin{aligned}
& \tau^{-1}=\tau_{0}^{-1} \exp \left(\frac{-U_{\text {eff }}}{T}\right) \\
& \tau^{-1}=C T^{n}+\text { QTM }
\end{aligned}
$$

\section{CONCLUSIONS}

Through systematic variable temperature single crystal X-ray diffraction studies, a coordination switch in the mononuclear
Co(II) complex 1 was revealed. Two distinct phases were identified-the high temperature $\alpha$ phase between 290 and $150 \mathrm{~K}$, which has a mixed coordination environment (95-75\% TBP and 5-25\% SPY), and the low temperature $\beta$ phase where 1 adopts a pentagonal bipyramidal coordination environment via an ordering of the nitrate ligands below 140 $\mathrm{K}$. The transition between the two phases was found to be reversible and robust. Complex 1 displays slow relaxation of the magnetization under an applied dc field, and the relaxation data can be accounted for by considering Raman processes and quantum tunneling of the magnetization. This study highlights the importance of conducting thorough low temperature investigations of the crystal structure, particularly where magnetic characterization is carried out at such low temperatures, with analysis reliant on the former. Note that if we had only measured the single-crystal structure at $\geq 150 \mathrm{~K}$, then the interpretation of the magnetic data would be for a trigonal bipyramidal $\mathrm{Co}$ (II) complex and therefore totally incorrect. Work is already underway to probe the coordination switching further through modification of the surrounding ligands (e.g., using $[\mathrm{MDABCO}]^{+}\left\{\mathrm{MDABCO}^{+}=1\right.$-methyl-4-aza-1azoniabicyclo[2.2.2] octanium cation $\}$ ) in order to understand the limits of this behavior, and to investigate the impact on the magnetic anisotropy of such systems further.

\section{ASSOCIATED CONTENT}

\section{Supporting Information}

The Supporting Information is available free of charge on the ACS Publications website at DOI: 10.1021/acs.inorgchem.9b00515.

Crystallographic data, crystal packing diagrams, information on SHAPE studies conducted, powder X-ray diffraction for complexes 1 and 2, and further magnetic data (PDF)

\section{Accession Codes}

CCDC 1897475-1897487, 1897499-1897504, and 1914822-1914823 contain the supplementary crystallographic data for this paper. These data can be obtained free of charge via www.ccdc.cam.ac.uk/data_request/cif, or by emailing data_request@ccdc.cam.ac.uk, or by contacting The Cambridge Crystallographic Data Centre, 12 Union Road, Cambridge CB2 1EZ, UK; fax: +44 1223 336033. The relevant data corresponding to this work are available at http://dx.doi.org/10.5525/gla.researchdata.846.

\section{AUTHOR INFORMATION}

\section{Corresponding Author}

*E-mail: mark.murrie@glasgow.ac.uk.

ORCID $\odot$

Mark Murrie: 0000-0001-7297-2878

Funding

M.M. thanks the UK Engineering and Physical Sciences Research Council for financial support (EPSRC EP/ N01331X/1 and EP/M508056/1).

\section{Notes}

The authors declare no competing financial interest.

\section{REFERENCES}

(1) Chang, E. L.; Simmers, C.; Knight, D. A. Cobalt Complexes as Antiviral and Antibacterial Agents. Pharmaceuticals 2010, 3, 17111728. 
(2) Di Giovanni, C.; Gimbert-Surinach, C.; Nippe, M.; BenetBuchholz, J.; Long, J. R.; Sala, X.; Llobet, A. Dinuclear Cobalt Complexes with a Decadentate Ligand Scaffold: Hydrogen Evolution and Oxygen Reduction Catalysis. Chem. - Eur. J. 2016, 22, 361-369.

(3) Sun, B.; Ou, Z.; Meng, D.; Fang, Y.; Song, Y.; Zhu, W.; Solntsev, P. V.; Nemykin, V. N.; Kadish, K. M. Electrochemistry and Catalytic Properties for Dioxygen Reduction Using Ferrocene-Substituted Cobalt Porphyrins. Inorg. Chem. 2014, 53, 8600-8609.

(4) Heffern, M. C.; Yamamoto, N.; Holbrook, R. J.; Eckermann, A. L.; Meade, T. J. Cobalt Derivatives as Promising Therapeutic Agents. Curr. Opin. Chem. Biol. 2013, 17, 189-196.

(5) Lunghi, A.; Totti, F.; Sessoli, R.; Sanvito, S. The Role of Anharmonic Phonons in Under-Barrier Spin Relaxation of Single Molecule Magnets. Nat. Commun. 2017, 8, 14620.

(6) Lunghi, A.; Totti, F.; Sanvito, S.; Sessoli, R. Intra-Molecular Origin of the Spin-Phonon Coupling in Slow-Relaxing Molecular Magnets. Chem. Sci. 2017, 8, 6051-6059.

(7) Escalera-Moreno, L.; Suaud, N.; Gaita-Ariño, A.; Coronado, E. Determining Key Local Vibrations in the Relaxation of Molecular Spin Qubits and Single-Molecule Magnets. J. Phys. Chem. Lett. 2017, $8,1695-1700$.

(8) Moseley, D. H.; Stavretis, S. E.; Thirunavukkuarasu, K.; Ozerov, M.; Cheng, Y.; Daemen, L. L.; Ludwig, J.; Lu, Z.; Smirnov, D.; Brown, C. M.; Pandey, A.; Ramirez-Cuesta, A. J.; Lamb, A. C.; Atanasov, M.; Bill, E.; Neese, F.; Xue, Z.-L. Spin-phonon Couplings in Transition Metal Complexes with Slow Magnetic Relaxation. Nat. Commun. 2018, 9, 2572.

(9) Collet, A.; Craig, G. A.; Heras Ojea, M. J.; Bhaskaran, L.; Wilson, C.; Hill, S.; Murrie, M. Slow Magnetic Relaxation in a $\{$ CoIICoIII2\} Complex Containing a High Magnetic Anisotropy Trigonal Bipyramidal CoII Centre. Dalton Trans. 2018, 47, 9237-9240.

(10) Schulte, K. A.; Vignesh, K. R.; Dunbar, K. R. Effects of Coordination Sphere on Unusually Large Zero Field Splitting and Slow Magnetic Relaxation in Trigonally Symmetric Molecules. Chem. Sci. 2018, 9, 9018-9026.

(11) Ruamps, R.; Batchelor, L. J.; Guillot, R.; Zakhia, G.; Barra, A.L.; Wernsdorfer, W.; Guihéry, N.; Mallah, T. Ising-Type Magnetic Anisotropy and Single Molecule Magnet Behaviour in Mononuclear Trigonal Bipyramidal Co(II) Complexes. Chem. Sci. 2014, 5, 34183424.

(12) Rechkemmer, Y.; Breitgoff, F. D.; van der Meer, M.; Atanasov, M.; Hakl, M.; Orlita, M.; Neugebauer, P.; Neese, F.; Sarkar, B.; van Slageren, J. A Four-Coordinate Cobalt(II) Single-Ion Magnet with Coercivity and a Very High Energy Barrier. Nat. Commun. 2016, 7, 10467.

(13) Hou, X.; Wang, X.; Liu, X.; Wang, J.-J.; Tang, L.; Ju, P. FineTuning the Effects of Auxiliary Ligands on Two Trigonal-Bipyramid Cobalt(II) Complexes Exhibiting Field-Induced Slow Magnetic Relaxation. New J. Chem. 2018, 42, 8583-8590.

(14) Mondal, A. K.; Jover, J.; Ruiz, E.; Konar, S. Quantitative Estimation of Ising-Type Magnetic Anisotropy in a Family of $C_{3}$ -Symmetric Co ${ }^{\mathrm{II}}$ Complexes. Chem. - Eur. J. 2017, 23, 12550-12558. (15) Rigamonti, L.; Bridonneau, N.; Poneti, G.; Tesi, L.; Sorace, L.; Pinkowicz, D.; Jover, J.; Ruiz, E.; Sessoli, R.; Cornia, A. A PseudoOctahedral Cobalt(II) Complex with Bis-Pyrazolylpyridine Ligands Acting as a Zero-Field Single-Molecule Magnet with Easy Axis Anisotropy. Chem. - Eur. J. 2018, 24, 8857-8868.

(16) Novikov, V. V.; Pavlov, A. A.; Nelyubina, Y. V.; Boulon, M.-E.; Varzatskii, O. A.; Voloshin, Y. Z.; Winpenny, R. E. P. A Trigonal Prismatic Mononuclear Cobalt(II) Complex Showing Single-Molecule Magnet Behavior. J. Am. Chem. Soc. 2015, 137, 9792-9795.

(17) Sertphon, D.; Murray, K. S.; Phonsri, W.; Jover, J.; Ruiz, E.; Telfer, S. G.; Alkas, A.; Harding, P.; Harding, D. J. Slow Relaxation of Magnetization in a Bis-Mer-Tridentate Octahedral Co(II) Complex. Dalton Trans. 2018, 47, 859-867.

(18) Ruamps, R.; Batchelor, L. J.; Guillot, R.; Zakhia, G.; Barra, A.L.; Wernsdorfer, W.; Guihéry, N.; Mallah, T. Ising-Type Magnetic Anisotropy and Single Molecule Magnet Behaviour in Mononuclear
Trigonal Bipyramidal Co(II) Complexes. Chem. Sci. 2014, 5, 34183424.

(19) Zadrozny, J. M.; Long, J. R. Slow Magnetic Relaxation at Zero Field in the Tetrahedral Complex $[\mathrm{Co}(\mathrm{SPh}) 4] 2-$ J. Am. Chem. Soc. 2011, 133, 20732-20734.

(20) Gómez-Coca, S.; Urtizberea, A.; Cremades, E.; Alonso, P. J.; Camón, A.; Ruiz, E.; Luis, F. Origin of Slow Magnetic Relaxation in Kramers Ions with Non-Uniaxial Anisotropy. Nat. Commun. 2014, 5, 4300.

(21) Murrie, M. Cobalt(II) Single-Molecule Magnets. Chem. Soc. Rev. 2010, 39, 1986-1995.

(22) Craig, G. A.; Murrie, M. 3d Single-Ion Magnets. Chem. Soc. Rev. 2015, 44, 2135-2147.

(23) Rosado Piquer, L.; Sañudo, E. C. Heterometallic 3d-4f SingleMolecule Magnets. Dalton Trans. 2015, 44, 8771-8780.

(24) Chakarawet, K.; Bunting, P. C.; Long, J. R. Large Anisotropy Barrier in a Tetranuclear Single-Molecule Magnet Featuring LowCoordinate Cobalt Centers. J. Am. Chem. Soc. 2018, 140, 2058-2061.

(25) Yao, X.-N.; Du, J.-Z.; Zhang, Y.-Q.; Leng, X.-B.; Yang, M.-W.; Jiang, S.-D.; Wang, Z.-X.; Ouyang, Z.-W.; Deng, L.; Wang, B.-W.; et al. Two-Coordinate $\mathrm{Co}$ (II) Imido Complexes as Outstanding Single-Molecule Magnets. J. Am. Chem. Soc. 2017, 139, 373-380.

(26) Fortier, S.; Le Roy, J. J.; Chen, C.-H.; Vieru, V.; Murugesu, M.; Chibotaru, L. F.; Mindiola, D. J.; Caulton, K. G. A Dinuclear Cobalt Complex Featuring Unprecedented Anodic and Cathodic Redox Switches for Single-Molecule Magnet Activity. J. Am. Chem. Soc. 2013, $135,14670-14678$.

(27) Hay, M. A.; Sarkar, A.; Craig, G. A.; Bhaskaran, L.; Nehrkorn, J.; Ozerov, M.; Marriott, K. E. R.; Wilson, C.; Rajaraman, G.; Hill, S.; et al. In-Depth Investigation of Large Axial Magnetic Anisotropy in Monometallic 3d Complexes Using Frequency Domain Magnetic Resonance and Ab Initio Methods: A Study of Trigonal Bipyramidal Co(II). Chem. Sci. 2019, 10, 6354-6361.

(28) Gómez-Coca, S.; Aravena, D.; Morales, R.; Ruiz, E. Large Magnetic Anisotropy in Mononuclear Metal Complexes. Coord. Chem. Rev. 2015, 289-290, 379-392.

(29) Farrell, A. R.; Coome, J. A.; Probert, M. R.; Goeta, A. E.; Howard, J. A. K.; Lemée-Cailleau, M. H.; Parsons, S.; Murrie, M. Ultra-Low Temperature Structure Determination of a Mn12 SingleMolecule Magnet and the Interplay between Lattice Solvent and Structural Disorder. CrystEngComm 2013, 15, 3423-3429.

(30) Viossat, B.; Benazeth, S.; Khodadad, P.; Rodier, N. Structure de l'aqua(Aza-4 Azonia-1 Bicyclo[2.2.2] Octane)Trinitratocobalt(II), [Co(C6H13N2)(NO3)3(H2O)]. Acta Crystallogr., Sect. C: Cryst. Struct. Commun. 1984, 40, 1340-1342.

(31) SAINT, 8.34A; Bruker AXS Inc.: Madison, WI, 2014.

(32) Sheldrick, G. M. SADABS, 2008-1 ed.; Bruker AXS Inc.: Madison, WI, 2008.

(33) Probert, M. R.; Robertson, C. M.; Coome, J. A.; Howard, J. A. K.; Michell, B. C.; Goeta, A. E. The XIPHOS Diffraction Facility for Extreme Sample Conditions. J. Appl. Crystallogr. 2010, 43, 14151418.

(34) McMonagle, C. J.; Probert, M. R. Reducing the Background of Ultra-Low-Temperature X-Ray Diffraction Data through New Methods and Advanced Materials. J. Appl. Crystallogr. 2019, 52, $445-450$.

(35) Dolomanov, O. V.; Bourhis, L. J.; Gildea, R. J.; Howard, J. A. K.; Puschmann, H. OLEX2: A Complete Structure Solution, Refinement and Analysis Program. J. Appl. Crystallogr. 2009, 42, 339-341.

(36) Sheldrick, G. M. SHELXT - Integrated Space-Group and Crystal-Structure Determination. Acta Crystallogr., Sect. A: Found. Adv. 2015, 71, 3-8.

(37) Macrae, C. F.; Edgington, P. R.; McCabe, P.; Pidcock, E.; Shields, G. P.; Taylor, R.; Towler, M.; Van De Streek, J. Mercury: Visualization and Analysis of Crystal Structures. J. Appl. Crystallogr. 2006, 39, 453-457.

(38) Pinsky, M.; Avnir, D. Continuous Symmetry Measures. 5. The Classical Polyhedra. Inorg. Chem. 1998, 37, 5575-5582. 
(39) Casanova, D.; Cirera, J.; Llunell, M.; Alemany, P.; Avnir, D.; Alvarez, S. Minimal Distortion Pathways in Polyhedral Rearrangements. J. Am. Chem. Soc. 2004, 126, 1755-1763.

(40) Alvarez, S. Distortion Pathways of Transition Metal Coordination Polyhedra Induced by Chelating Topology. Chem. Rev. 2015, 115, 13447-13483.

(41) Huang, X.-C.; Zhou, C.; Shao, D.; Wang, X.-Y. Field-Induced Slow Magnetic Relaxation in Cobalt(II) Compounds with Pentagonal Bipyramid Geometry. Inorg. Chem. 2014, 53, 12671-12673.

(42) Shao, D.; Zhang, S.-L.; Shi, L.; Zhang, Y.-Q.; Wang, X.-Y. Probing the Effect of Axial Ligands on Easy-Plane Anisotropy of Pentagonal-Bipyramidal Cobalt(II) Single-Ion Magnets. Inorg. Chem. 2016, 55, 10859-10869.

(43) Mondal, A. K.; Mondal, A.; Dey, B.; Konar, S. Influence of the Coordination Environment on Easy-Plane Magnetic Anisotropy of Pentagonal Bipyramidal Cobalt(II) Complexes. Inorg. Chem. 2018, 57, 9999-10008.

(44) Chen, L.; Chen, S.-Y.; Sun, Y.-C.; Guo, Y.-M.; Yu, L.; Chen, X.T.; Wang, Z.; Ouyang, Z. W.; Song, Y.; Xue, Z.-L. Slow Magnetic Relaxation in Mononuclear Seven-Coordinate Cobalt(II) Complexes with Easy Plane Anisotropy. Dalton Trans. 2015, 44, 11482-11490.

(45) Woods, T. J.; Ballesteros-Rivas, M. F.; Gómez-Coca, S.; Ruiz, E.; Dunbar, K. R. Relaxation Dynamics of Identical Trigonal Bipyramidal Cobalt Molecules with Different Local Symmetries and Packing Arrangements: Magnetostructural Correlations and $\mathrm{Ab}$ Inito Calculations. J. Am. Chem. Soc. 2016, 138, 16407-16416.

(46) Fernández de Luis, R.; Larrea, E. S.; Orive, J.; Lezama, L.; Arriortua, M. I. Commensurate Superstructure of the $\{\mathrm{Cu}(\mathrm{NO} 3)$ $(\mathrm{H} 2 \mathrm{O})\}(\mathrm{HTae})$ (Bpy) Coordination Polymer: An Example of 2D Hydrogen-Bonding Networks as Magnetic Exchange Pathway. Inorg. Chem. 2016, 55, 11662-11675.

(47) Chilton, N. F.; Anderson, R. P.; Turner, L. D.; Soncini, A.; Murray, K. S. PHI: A Powerful New Program for the Analysis of Anisotropic Monomeric and Exchange-Coupled Polynuclear d- and fBlock Complexes. J. Comput. Chem. 2013, 34, 1164-1175.

(48) Deng, Y.-F.; Yao, B.; Zhan, P.-Z.; Gan, D.; Zhang, Y.-Z.; Dunbar, K. R. Synthesis and Magnetic Studies of Pentagonal Bipyramidal Metal Complexes of Fe, Co and Ni. Dalton Trans. 2019, 48, 3243-3248.

(49) Trachenko, K. Understanding the Spin Glass Transition as a Dynamic Phenomenon. J. Phys.: Condens. Matter 2011, 23, 366003.

(50) Bałanda, M. AC Susceptibility Studies of Phase Transitions and Magnetic Relaxation: Conventional, Molecular and Low-Dimensional Magnets. Acta Phys. Pol., A 2013, 124, 964-976.

(51) Cole, K. S.; Cole, R. H. Dispersion and Absorption in Dielectrics I. Alternating Current Characteristics. J. Chem. Phys. 1941, 9, 341 .

(52) Guo, Y.-N.; Xu, G.-F.; Guo, Y.; Tang, J. Relaxation Dynamics of Dysprosium(III) Single Molecule Magnets. Dalton Trans. 2011, 40, 9953-9963.

(53) Vallejo, J.; Castro, I.; Ruiz-García, R.; Cano, J.; Julve, M.; Lloret, F.; De Munno, G.; Wernsdorfer, W.; Pardo, E. Field-Induced Slow Magnetic Relaxation in a Six-Coordinate Mononuclear Cobalt(II) Complex with a Positive Anisotropy. J. Am. Chem. Soc. 2012, 134, 15704-15707.

(54) Wu, D.; Zhang, X.; Huang, P.; Huang, W.; Ruan, M.; Ouyang, Z. W. Tuning Transverse Anisotropy in CoIII-CoII-CoIII MixedValence Complex toward Slow Magnetic Relaxation. Inorg. Chem. 2013, 52, 10976-10982.

(55) Zadrozny, J. M.; Liu, J.; Piro, N. A.; Chang, C. J.; Hill, S.; Long, J. R. Slow Magnetic Relaxation in a Pseudotetrahedral Cobalt(II) Complex with Easy-Plane Anisotropy. Chem. Commun. 2012, 48, 3927-3929.

(56) Carlin, R. L. Magnetochemistry; Springer-Verlag: Berlin, 1986.

(57) Gatteschi, D.; Sessoli, R. Quantum Tunneling of Magnetization and Related Phenomena in Molecular Materials. Angew. Chem., Int. Ed. 2003, 42, 268-297.

(58) Shrivastava, K. Theory of Spin-Lattice Relaxation. Phys. Status Solidi B 1983, 117, 437-458.
(59) Singh, A.; Shrivastava, K. Optical-acoustic Two-phonon Relaxation in Spin Systems. Phys. Status Solidi B 1979, 95, 273-277. (60) Santillan, G. A.; Carrano, C. J. Synthesis and Characterization of Copper (II) Complexes of Nonfacially Coordinating Heteroscorpionate Ligands Synthesis and Characterization of Copper (II) Complexes of Nonfacially Coordinating Heteroscorpionate Ligands. Inorg. Chem. 2007, 46, 1751-1759.

(61) Abragam, A.; Bleaney, B. Electron Paramagnetic Resonance of Transition Ions; Oxford University Press, 1970. 